\section{Comparing the Australian national health system with the Vietnamese national health system from the perspective of future challenges}

\author{
Van Nguyen \\ Senior Clinical Pharmacist, Department \\ of Pharmacy, The Royal Melbourne \\ Hospital, Australia
}

\section{Correspondence: Van Nguyen}

\section{Abstract}

Background: Performance of a health system can be accessed using the primary goals of responsiveness to the expectation of the population, financial contribution fairness and good health. Results depend fundamentally on how well systems carry out four vital functions: financing, resource generation, stewardship, and service provision.

Aim: To analyse and compare the Australian national health system with the Vietnamese national health system from the perspective of future challenges such as chronic disease, advancing information technologies and expenditure. To discuss the implications for health policy considering social, environmental and economic factors for each country.

Method: A systematic literature search was undertaken using the literature databases Medline OVID, Embase and PubMed, accessed through the Southern Cross University (SCU) and The Royal Melbourne Hospital library websites. Key terms: 'Australian healthcare system', 'Vietnam Healthcare system', 'National health system', were identified. When broadened, other key terms were used in database to optimize the search strategies. The search was restricted to English articles published between 1949 to the present. Reference reading list and online weekly content from SCU discussion forum, when combined with the results of the literature search, provided robust resources to compare Australia's national health system with Vietnam's health system from the perspective of future challenges.

Conclusion: Performance of health systems has been a major concern of policy makers for many years. Health systems cannot afford to only focus on improving people's health alone but also protecting them against the burden of financial costs of illness. The challenge facing policy makers of low income countries, such as Vietnam, is to reduce out-of-pocket payment for health by developing prepayment schemes, which is intended to expand financial risk and reduce the spectre of on-going health payments.

Keywords: Australian health system, Vietnam health system, health policy

Received: December 24, 2016, Accepted: January 10, 2017, Published: January 17, 2017

\section{Introduction}

Performance of health systems is a major concern for decision makers for many years. Many countries have recently introduced reforms in the health sector with the explicit aim of improving performance [1]. Measurement of performance requires detailed frameworks, defining the goals of a health system against which outcomes can be judged and performance quantified [2]. Over the last decade, health across the world has improved, as reflected by improvements in key indicators such as life expectancy at birth, disability-adjusted life expectancy or mortality amenable to health care [3]. With these improvements come on-going concerns relating to future challenges. These challenges include the increasing prevalence of chronic diseases, variations in the 
quality and safety standards, inequalities between and within countries in access to care and health outcomes, an incongruity between health needs and human resources, and rising health care cost [1]. It is vital that country invest in well-functioning health systems. It is the role of governments to focus on healthcare improvements and efficiency gains [4].

The World Health Organization has carried out the first ever analysis of 191 countries' health systems in 2000 [5]. Health system performance was accessed in view of the primary goals of good health, responsiveness to the expectation of the population and fairness of financial contribution. Progress towards them depends on how well systems carry out four vital functions: service provision, resource generation, financing and stewardship [2]. Australia was ranked 32 while Vietnam was ranked 160 [5]. The results of this report, quashed the misconception that financial expenditure alone can correlate to a health system's performance, disputing a common argument that the more money a health system has the better the system should function. Power house countries like America and the United Kingdom spend significant portion of its gross domestic product on healthcare, ranked much lower than smaller countries like San Marino and Singapore, who do not have the wealth of these bigger countries [5]. While the configuration of services varies from country to country, common elements of chronic disease, funding, a skilled workforce, reliable information on which to base decisions and policies, and wellmaintained facilities and logistics to deliver quality medicines and technologies, prove all similar in present and future implications [3].

This report attempts to analyse and compare the Australian national health system with the Vietnamese national health system from the perspective of these future challenges.

\section{The Australian Healthcare System}

Australia spends more on its healthcare every year, even after concessions for the national inflation rate. As a proportion of all spending on goods and services, health spending has increased from $7.9 \%$ to $9.4 \%$ over the past decade. The out-of-pocket health expenditure for Australian in 2014 was 18.8\%. Australia spent $\$ 121.4$ billion on health in 2009-10, which accounted for $9.4 \%$ of total spending on all goods and services in the economy (known as gross domestic product or GDP). This averaged out to $\$ 5,479$ per person [6]. The difficult task for governing bodies is to provide accessible healthcare to over 24 million people spanning over an area of 7.692 million $\mathrm{km}^{2}$ [6].

The Australian health system is a network private and public institution, in almost all areas of the nation, supported by a multi-facet complex network. Health providers including doctors, nurses, and allied health professionals, deliver essential services across many levels, to the community, in the form of primary health care, hospital-based and emergency treatment [1].

\section{The National Public Health Insurance Scheme: Medicare is the primary Australian}

Government's funding contribution to healthcare in this country. Medicare was introduced in 1984 to provide free or subsidised treatment by health professionals to patients. The Medicare system may be separated into three sections: hospital, medical and pharmaceutical. Medicare is design to include free treatment for patients in all public hospitals, reimbursement or rebates for services provided by health professionals/institutions and subsidisation of the costs of certain prescription medicines listed under the Pharmaceutical Benefits Scheme [7].

Although the program is designed to provide free healthcare, it does not include an array of arguably essential services such as ambulance and most dental services, occupational therapy, podiatry, most physiotherapy, speech therapy, and psychology services. The use of medical aids such as glasses, contact lenses, hearing aids and other appliances are also not included and patients are expected to absorb these cost themselves [8].

As the system moves towards exceeding three decades in motion, it faces new challenges. Chronic disease represents a significant challenge to the design and reform of the Australian healthcare system. The Medicare Benefits Schedule (MBS) provides a framework of numerous chronic disease management programs, but the multitude of available programs and items are administratively complex, overlapping and subject to claiming incompatibilities [9]. Providing patients with optimum chronic disease management requires both clinical and administrative skill on the part of GPs. Time spent on administrative requirements is time away from clinical care. Although quality improvement efforts may improve functioning within the existing system, broader system reforms is essential to support optimal chronic disease management in Australia [10].

\section{The Vietnamese Healthcare System}

Vietnam, a communist country since 1975 , embarked on a course of increased economic liberalisation and structural reforms to modernise its economy in the mid 80's. By 2010 Vietnam had advanced from its previous status as a low-income country to become a lower- middle-income country [11]. The advancement in financial status enabled governing parties to invest in the health care system. Vietnam's private and public health system is focused on achieving universal, affordable coverage to almost 90 million people across the country the size of approximately $330,000 \mathrm{~km}^{2}$ [12], but is currently yet to achieve this vision. The Vietnamese economy has been growing at sustainable speed thanks to rational measures. The national economic growth rate stays at $6-7 \%[12]$.

Rapid and sustainable economic growth has facilitated favourable conditions for increasing investment in health and health promotion. Vietnam reports a GDP of $6.2 \%$. This averages to an allocation of $\$ 2690$ per person. Public expenditure on health takes $11.0 \%$ of total annual state expenditure but they have a high out-of-pocket expenditure rate of $36.8 \%$ for healthcare services [13]. These figures make it financially challenging for Vietnam to make significant gains in terms of performance. The public system is organised under an administrative hierarchy, with the provincial health authorities sitting below the Ministry of Health [14]. Although remarkable gains of improvements in mortality rates of infants and under-five child, there remain significant inequalities in access to health care and health outcomes across the country [15]. Excessive personal expenditure coupled with 
ongoing health scandals contribute to a general distrust of the system and reluctance for change [16]. Although, there are many deficiencies in their system there are positive aspects that warrant recognition. Immunizations, hygiene, nutrition, maternal/paediatric healthcare are provided without cost and recent government policy of rotation in certain places for specific doctors to serve in underserved areas has become compulsory [17]. Another challenge faced by the system is that patients prefer to seek care at provincial/central hospitals for common treatable diseases that can easily manageable at a district/communal level. Bypassing in health care can lead to congestion in central areas and under usage of services in other areas [17]. This causes avoidable wastage for the entire health care system.

Health system technology development has shown considerate improvements in recent years. Many legal policies in health information have been issued, including Law on Statistics, the program for national surveys and health indicator system. Unfortunately, in health information systems there are still significant deficiencies. Currently no policy, orientation, and health information system development plan have been drafted [14]. The country's policies for standard treatment protocols, guidelines for diagnosis and treatment of diseases, and care pathways are only restricted to only some diseases [16]. This shortfall is a significant gap for policy makers but still, government agencies do not appear to invested sufficient time and resources to prioritise this deficiency. The system does not support continuity of care. There is no transparent universal referral or tracking of patient data which affects the quality of overall healthcare and compounds treatment costs due to possible duplication [18]. Collaboration mechanism between agencies, departments within the health sector and external institutions tend to be uncooperative. Multiple contributing factors to this breakdown include limited knowledge in data analysis, excessive personnel involvement poor database, shortage facilities, failure to manage and update information, store and transmit data by modern technology [15]. Like most countries, Vietnam also faces challenges with regards to burden of chronic diseases. The current disease pattern of Vietnam is in a transitional period. Although infectious diseases have declined, some communicable diseases are still at risk of reoccurring (e.g., Cholera, dengue fever). The trend has also indicated that the highest burden in Vietnam in 2006 has moved to complaints such as cardiovascular diseases and mental illness [12]. This increase in these chronic conditions has lead to escalating health care costs. The average treatment cost is considerably higher for these issues as it requires high technologies, increased service costs, expensive specific medicines, additional complications and longer treatment periods. This additional challenge for the Vietnamese health care system coupled with weak policy implementation, makes the health system performance extremely poor.

\section{Comparison of the Two Systems}

Social, environmental, and economic factors bare significant influence on a country's healthcare system. When comparing these two health systems there are substantial differences that result in Australia's health system out performing that of the Vietnamese system. To demonstrate these comparisons, the issue of medication supply, water sanitation and socioeconomically disadvantaged will be discussed.

\section{Prescription pharmaceuticals}

Both countries have national policies on the provision of pharmaceuticals and sufficient access to drugs, thanks to a widespread network of drug distribution throughout the country. In Australia, the provision of pharmaceuticals is reimbursed under the national pharmaceutical benefit scheme (PBS). Under the PBS, Australians pay only part of the cost of most prescription medications. The PBS covers the rest of the cost [7]. Medication provided in the setting as an in-patient in public hospitals are free to all patients, with the cost covered by state and territory governments. Unfortunately, Vietnam has failed to widely apply appropriate payment methods. Fake medicines, poor quality drugs including eastern medicine and pharmaceutical materials remain an ongoing worry for decision makers [14]. Strengthening drug quality control work in terms of number of staff and professional capacity is essential to overcome this issue. However, the state budget is allocated to purchase only some essential drugs in the national target programs and free dispensary of drugs is only for patients with certain special diseases (TB, HIV/AIDS infected patients, schizophrenia, epilepsy) [15].

The administration and control of drug prices in Vietnam market remains a big challenge. Drug price in Vietnam is higher than international reference price, including generic and specialized drugs. Tender in drug procurement seems ineffective in reducing drug price. Some drugs have very limited registered quotas, which creates monopoly and price increase in some drugs [16]. Inversely, in Australia there is The Therapeutic Goods Administration (TGA), which is a governing body responsible for regulating therapeutic goods including prescription medications and vaccines. It is an external link to part of Department of Health [19]. Almost any product for which therapeutic claims are made must be entered in the Australian Register of Therapeutic Goods (ARTG) and abide by strict standards, before it is made available for supply in Australia. Service provision in the form of prescription pharmaceuticals is thus highly advantageous in Australia compared to that seen in Vietnam.

\section{Water sanitation}

Water sanitation and hygiene is essential for people's health and quality of life. The environmental health issue to a country that does not supply adequate water to its people is colossal. Poor sanitation leads to premature deaths globally each year, with increasing number of children dying from preventable diarrhoeal disease and dehydration [13]. Inadequate access to water sanitation and hygiene is associated with the spread of diseases including polio, hepatitis and cholera [13]. Australia's water supply and sanitation is clean and universal. Water conservation and various regional restrictions on the use of water is occasionally implemented in vulnerable drought areas and dependent on the current climate. In a 2015 report, it was documented that Vietnam has $98 \%$ of Vietnamese residents have access to improved drinking water sources and $78 \%$ of 
the population uses toilets and latrines that meet international standards [13]. Although this result is not universal, merit should be noted as these figures were $87 \%$ and $54 \%$ respectively in 2009 [12]. The provision of water sanitation concludes Australia out performs Vietnam in this comparison.

\section{Socio-economic factor}

The socioeconomically disadvantaged provides a unique challenge to healthcare services and professionals as they are affected holistically [20]. This means the physical, mental, social, financial and emotional health of the population contributes to barriers as these patients are less likely to access health systems, especially preventative services such as screening. They are also more likely to exhibit common health risk factors. There is a strong correlation between socioeconomically disadvantage and healthcare outcomes [21]. Socioeconomically disadvantage factors include income, education, occupation, health beliefs and place of residence. The Australia's Health 2014 report by the AlHW states that the death rate is higher amongst those from a lower socioeconomic status (SES) for both genders of all age groups. Not only do Australians from the lower SES groups have a higher death rate, they also have a higher disability rate. They smoke more and suffer from chronic diseases like depression, type 2 diabetes, and cardiovascular disease [6]. This is similarly mirrored in the Vietnamese population and contributes to the same health outcome concerns associated with this population group in this country.

Dispute a significant difference in both national health systems there appears to be similarity in the future challenges for both countries. This includes access to healthcare in regional areas and the provision of adequate skilled workforce. Lack of personnel is one of the most important constraints to strengthening the delivery of primary and secondary health services, including preventive and rehabilitative services. Both countries face concerns with the existence of an imbalanced distribution of health workforce, scarce health workers in some specialties and rural areas. Health workforce with high qualifications tends to concentrate in urban and major cities. Migration of health workforce away from lower to higher level, rural to urban and from public to private sector and high level facilities tends to be the trend, which affects secured availability of health workers in rural and remote areas.

\section{Implications for health policy}

Obstacles including geographical barriers, ageism, the poor preparedness of health systems to deliver age-appropriate care for chronic diseases, and the complexity of integrating care using multifaceted technological systems, poses significant implications for health policy. Both countries require exploration in innovations such as personally controlled electronic health (e-health) records and telehealth. These systems offer improved communication and access to services. An e-health record allows patients, medical staff, hospitals, and other health-care providers to view and share the patient's health information, with patients' prior consent [18]. Information of complete medication therapy, hospital discharge records, allergies, and immunisations may all be appropriate stored and disclosed when appropriate to advance health care performance. All these systems are expensive and require detailed strategic planning and significantly relies on health policy for successful implementation. Telehealth services use communication technologies, such as video-conferencing, to deliver health services and transmit health information. Telehealth technology can improve access to services for people living in regional, rural and remote areas [22]. Patients who travel extensively for consultation now have the option of using video-conferencing, provided it was offered at their local GP or another local health-care venue. These services also improve health system performance but will be limited to the effectiveness of adequate health policy planning. Responsiveness of the health sector to these issues is usually slow. Many health facilities are deteriorated, often medical equipment is out-dated and asynchronous. Reform policies and mechanisms are often delayed and this is a significant barrier to progress. Improving the health of older people and encouraging active and positive ageing are priorities for governments of all countries at all governing levels. Several strategies and frameworks support the concept of healthy ageing [23]. In Victoria, the state government is heavily invested in the provision of aged residential care, with the state owning and operating through publicly funded agencies $16 \%$ of residential aged care facilities [24]. Many of these facilities are in rural Victoria. The state government funds redevelopment and construction projects of facilities, and contributions for specialised care equipment, workforce recruitment and training and trialling of alternative care models [25].

Health systems must take into consideration the population and area to which healthcare services must be provided. Vietnam with almost 90 million people living in approximately $330,000 \mathrm{~km}^{2}$ will need to target their policies with high dense living environments and mountainous landscape compared to Australia's, 24 million people living in an area of 7.692 million $\mathrm{km}^{2}$, having to consider accessibility and coverage as factors. Policies need ongoing reviewed to reflect ongoing change of these parameters to meet the needs of the people while maintaining affordability of healthcare provision $[26,27]$.

\section{Conclusion}

When comparing the Australian health system to Vietnamese health system, there are clear benefits Australian have above their international brothers in relation to healthcare. Although there are obvious differences, both systems have the same future challenges of an ageing population presenting with chronic disease under a rapidly advancing technological environment. Structured health policy and strict health standards seen in Australian contributed to our health system outperforming that seen in Vietnam, ongoing investment of these strategies will aid any health systems to tactical many future challenges. Health systems do not solely focus on improving people's health alone but with protecting people against the financial costs of illness. The challenge facing government of low income countries is to reduce the regressive burden of out-of-pocket payment for health by expanding prepayment schemes, which spread financial risk and reduce the spectre of expanding health expenditures.

\section{Competing and Conflicting Interests}

Nil to declare. 


\section{References}

1 Bergstrom A, Skeen S, Duc DM, Blandon EZ, Estabrooks C, et al. (2015) Australian Government. Department of Human Services website.

2 Robbins A (2001) The World Health Report 2000: Health Systems: Improving Performance 116: 268-269.

3 Veillard JHM, Brown AD, Bar E, Permanand G, Klazinga NS, et al. (2011) Health system stewardship of National Health Ministries in the WHO European region: Concepts, functions and assessment framework. Health policy 103: 191-199.

4 Frenk J (2010) The global health system: strengthening national health systems as the next step for global progress. PLOS Medicine 7: e1000089.

5 World Health Organisation: WHO (2000) World Health Report 2000Health systems: improving performance. Geneva.

6 AlHW (2014) Australia's health 2014 : The 14th biennial welfare report of the Australian Institute of Health and Welfare.

7 PBS (2016) Department of Health: Pharmaceutical Benefits Scheme.

8 Gibbons E, Abc Ltd, I. T. V. S. A. P, Screen A, Australian Film C, Film Australia Pty L, Film Finance Corporation A (2016) Keeping Australia alive : our health system in a day. Australia: ITV Studios Screen Australia.

9 Grant C (1993) The Australian health care system 1992. Kensington, N,S.W: School of Health Services Management, University of New South Wales.

10 Taylor M, Swerissen H (2010) Medicare and Chronic Disease Management: Integrated Care as an Exceptional Circumstance? Australian Health Review 34: 152-161.

11 Landgraf KM, Kakkar R, Meigs M, Jankauskas PT, Phan TT, et al. (2016) Open-source LIMS in Vietnam: The path toward sustainability and host country ownership. Int J Med Inform 93: 92-102.

12 Ministry of Health M (2010) Five-Year Health sector Development plan 2011-2015: Hanoi.

13 World Health Organisation: WHO (2015) Progress on Sanitation and Drinking Water: 2015 Update and MDG Assessment.

14 Minh HV, Giangle M, Cashin C, Hinh ND (2015) Health system research in Vietnam: generating policy-relevant knowledge. Glob Public Health 10: S14.
15 Phuong ZK, Oanh TT, Phuong HT, Tien TV, Cashin C, et al. (2015) Assessment of systems for paying health care providers in Vietnam: implications for equity, efficiency and expanding effective health coverage. Glob Public Health 10: S80-94.

16 Thi Thu Ha B, Mirzoev T, Morgan R (2015) Patient complaints in healthcare services in Vietnam's health system. SAGE Open Med 3: 2050312115610127.

17 Tran BX, Nguyen LH, Nong VM, Nguyen CT (2016) Health status and health service utilization in remote and mountainous areas in Vietnam. Health Qual Life Outcomes 14: 85.

18 Nguyen L, Bellucci E, Nguyen LT (2014) Electronic health records implementation: An evaluation of information system impact and contingency factors. Int J Med Inform 83: 779-796.

19 Therapeutic Goods Administration: TGA (2016) Therapeutic Goods Administration Website.

20 Smith SA, Mays GP, Felix HC, Tilford JM, Curran GM, et al. (2015) Impact of economic constraints on public health delivery systems structures. 105: e48.

21 Friesen CE, Seliske P, Papadopoulos A (2016) Using Principal Component Analysis to Identify Priority Neighbourhoods for Health Services Delivery by Ranking Socioeconomic Status. Online J Public Health Inform 8: e192.

22 Colin Carati, George Margelis (2013) Towards a national strategy for telehealth in Australia 2013-2018. Australasian Telehealth Society.

23 Kendig H, Browning C (2011) Directions for ageing well in a healthy Australia. Dialogue 31: 23-30.

24 Prince MJ, Wu F, Guo Y, Gutierrez Robledo LM, et al. (2015) The burden of disease in older people and implications for health policy and practice. The Lancet 385: 549-562.

25 Vic-Health (2016) Ageing and aged care.

26 Armstrong B, Gillespie J, Leeder S, Rubin G, Russell L, et al. (2007) Challenges in health and health care for Australia. Medical Journal of Australia 187: 485-489.

27 Wallin L (2015) Health system context and implementation of evidence-based practices-development and validation of the Context Assessment for Community Health ( $\mathrm{COACH}$ ) tool for lowand middle-income settings. Implement Sci 10: 120. 